\title{
ESTIGMA HACIA PACIENTES PSIQUIÁTRICOS EN ESTUDIANTES MEXICANOS DE PSICOLOGÍA
}

\section{STIGMA TOWARDS PSYCHIATRIC PATIENTS IN MEXICAN PSYCHOLOGY STUDENTS}

Alma Alberta Martínez Castillo, Roberto Lagunes Córdoba, Francisco Javier Rosas Santiago

\author{
Universidad Veracruzana, México
}

Correspondencia: frosas98@yahoo.com.mx

\section{RESUMEN}

El objetivo de estudio fue analizar el estigma hacia la enfermedad mental en estudiantes de psicología, para determinar si los conocimientos y actitudes tienen valor predictivo sobre las conductas estigmatizantes hacia personas con alguna enfermedad mental. Participaron 864 universitarios mexicanos de tres diferentes entidades federativas, con una edad promedio de $21.4 \pm 4.2$ años, el $72.5 \%$ fueron mujeres. El estudio contó con la aprobación de un Comité de Ética y los datos se recabaron en las escuelas donde estudiaban los participantes. Se adaptaron al español para su aplicación tres cuestionarios de autorreporte: la Escala de Conocimiento de la Salud Mental (MAKS), la Escala de Actitudes de los Médicos (MICA) y la Escala de Comportamiento Informado e Intencionado (RIBS). Los resultados de este estudio mostraron que los estudiantes de psicología de semestres superiores y con mayor edad tuvieron mejores actitudes hacia la enfermedad mental. Además, el conocimiento, las actitudes y la experiencia de convivir con algún paciente psiquiátrico, son predictores de menos conductas de discriminación. Se discuten los hallazgos a la luz de sus implicaciones para la formación de los futuros psicólogos. 
Palabras clave: conocimiento, actitudes, discriminación, enfermedad mental, universitarios.

\section{ABSTRACT}

The objective of the study was to analyze stigma towards mental illness in psychology students, to determine whether knowledge and attitudes have a predictive value for stigmatizing behaviors towards people with a mental illness of some kind. Eight hundred and sixty-four Mexican university students from three different states participated, with an average age of $21.4 \pm 4.2$ years, $72.5 \%$ of whom were women. The study was approved by an ethics committee and the data were collected in the schools where the subjects were studying. Three self-report questionnaires were translated into Spanish for their administration: The Mental Health Knowledge Scale (MAKS), The Mental Illness Clinicians' Attitudes Scale (MICA) and The Reported and Intended Behavior Scale (RIBS). The results of this study showed that older psychology students in higher semesters had better attitudes toward mental illness. Moreover, knowledge, attitudes, and the experience of living with a psychiatric patient were found to be predictors of fewer discriminatory behaviors. The findings are discussed considering their implications for the training of future psychologists.

Key words: knowledge, attitudes, discrimination, mental illness, university students

\section{INTRODUCCIÓN}

Según la Organización de las Naciones Unidas (ONU, 2005) aproximadamente 450 millones de personas en todo el mundo sufren algún trastorno mental (TM), una de cada 4 personas padecerá de algún TM en alguna fase de su vida y solo un porcentaje reducido de ellas recibirá tratamiento. 
Con frecuencia, los derechos esenciales de los pacientes psiquiátricos no son respetados en su totalidad, debido a la presencia de estereotipos negativos de la población general hacia ellos, e incluso del personal sanitario, lo que obstaculiza el acceso a los servicios de salud y por tanto sus procesos de recuperación (ONU, 2018).

Los pacientes psiquiátricos se ven expuestos a situaciones de rechazo por parte de las personas con las que se relacionan. Este fenómeno ha suscitado un creciente interés en estudiosos de la conducta por comprender la interacción entre la población general y quienes padecen alguna enfermedad mental grave (EMG). En este sentido, se ha descrito teóricamente que la combinación de la falta de conocimiento (ignorancia y desinformación), las actitudes negativas (prejuicio) y los comportamientos excluyentes o de evitación (discriminación), son factores que en su conjunto constituyen el estigma en el contexto de la enfermedad mental (EM) (Thornicroft, 2008).

Reportes de investigación han indicado que los pacientes psiquiátricos son estigmatizados por el medio social al que pertenecen (Zárate, Ceballos, Contardo y Florenzano, 2006; Nord, Rössler, Lauber y Schizophr, 2006; López et al., 2010), por el círculo familiar en el que viven (Marques, Barbosa y Queiros, 2010; OMS, 2013; Thornicroft, Wyllie, Thornicroft y Mehta, 2013), por parte de amigos y compañeros de trabajo (Pederson, 2005) e incluso por el personal de salud que los atiende (Pederson, 2005; Nord, Rössler, Lauber y Schizophr, 2006; Thornicroft, 2008; Wright, Henderson, Thornicroft, Sharac y McCrone, 2015).

Estas conductas de discriminación hacia los pacientes psiquiátricos tienen consecuencias peores que los síntomas físicos de la enfermedad misma (Thornicroft et al., 2016), ya que al experimentar rechazo por el personal 
médico, las personas con algún malestar mental no buscan tratamiento o bien no lo cumplen adecuadamente (Ahmedani, 2011). Además, el temor al rechazo por parte del personal de salud lleva al paciente a demorar por años la búsqueda de atención (Hasan y Thornicroft, 2018). Este tiempo de espera entre el inicio de la sintomatología y llegar al tratamiento puede ser de 15 años en promedio para los trastornos del afecto, 25 años para trastornos del impulso y ansiedad y más de 25 años en el caso de abuso de sustancias (Medina, Real y Amador, 2015).

Si bien este panorama pone a los profesionales de la salud en un papel relevante en el proceso de estigmatización del paciente psiquiátrico, la investigación al respecto es relativamente nueva (Chin y Balon, 2006; Navarro y Trigueros, 2019). Asimismo, la mayoría de los estudios sobre este tema se han llevado a cabo con muestras de personal de salud que radican en países europeos y anglosajones y son pocas las investigaciones que se han realizado en Latinoamérica (Polanco-Frontera et al., 2013).

Diversos estudios han encontrado que las actitudes de estigma y rechazo por parte del personal de salud está relacionado con el contacto que han experimentado a lo largo de la vida con algún paciente psiquiátrico (Ay, Save y Fidanoglu, 2006; Senra-Rivera, Arriba-Rossetto, y SeoanePesqueira, 2008; Gilaberte, Failde, Salazar, y Caballero, 2012; MedinaMesa., Cruces-Montes, Merchán-Clavellino, Romero-Moreno y GuilBozal, 2019), y en algunos casos con los procesos de educación formal a los que han tenido acceso (Fresán et al., 2012; Medina-Mesa et al., 2019; Magliano et al., 2012; Poreddi, Thimmaiah y BadaMath, 2017). No obstante, aunque médicos, enfermeras y psicólogos, han recibido formación académica en el ámbito de la salud y EM, esto no necesariamente se ha traducido en mejores actitudes hacia pacientes psiquiátricos al momento de brindarles atención. 
Aunque las actitudes hacia la EM son menos negativas en estudiantes de ciencias de la salud que en estudiantes de otras carreras, el estigma hacia los pacientes psiquiátricos es un fenómeno que sigue presente en esta población (Medina-Mesa et al., 2019). Diversos estudios han documentado la presencia de juicios estigmatizantes y actitudes negativas hacia personas con EM en los futuros profesionales sanitarios (Ay et al., 2005; Querido, Tomas y Carvalho, 2016; Poreddi et al., 2017; Fresán et al., 2012). Sin embargo, la mayoría de los estudios al respecto han analizado los datos combinando muestras de estudiantes de diversas carreras, dejando abierta la posibilidad de realizar investigación al respecto en estudiantes de profesiones determinadas.

Considerando la importancia que tiene la formación académica en el desarrollo de actitudes que determinan la relación entre el personal de salud y los pacientes (Magliano et al., 2012), es importante identificar y evaluar la presencia de este tipo de estigma entre estudiantes de la salud (Polanco-Frontera et al., 2013). Conocer la expresión de esta variable en el medio universitario, podría facilitar la prevención de la discriminación hacia usuarios de los servicios de salud diagnosticados con alguna EM por parte de los futuros trabajadores sanitarios.

Aunque las investigaciones respecto al estigma hacia las personas con alguna EM se han dirigido a los estudiantes de psicología, estas han explorado el estigma desde la medición de actitudes (Bannatyne, y Stapleton, 2016; Fresán et al., 2012; Medina-Mesa, 2019; Meru, 2017; Petkari, 2017; Pingani, Coriani, Galeazzi, Nasi, y Franceschini, 2020; Zaninotto, Qian, Bassi, Solmi, y Salcuni, 2018), o la distancia social (Pranckeviviene, et al., 2018; Tillman, 2018), estudiando el estigma únicamente desde uno de sus posibles elementos y no como un constructo integrado en un esquema 
teórico coherente que permita una comprensión integral del estigma considerando las actitudes, conocimientos y conductas. Por ello, la presente investigación se realizó bajo la perspectiva teórica de Thornicrof (2008).

Realizar este tipo de estudios permite analizar las variables psicológicas específicas que dan como resultado el estigma, lo que podría traducirse en hallazgos que permitan un abordaje específico para la modificación de este fenómeno. Un estudio realizado con este enfoque teórico adquiere relevancia en muestras de futuros psicólogos si se toma en consideración que se trata de una profesión involucrada en la detección, tratamiento, rehabilitación y prevención de los TM no solo en el ámbito clínico, sino también en el laboral, educativo y comunitario. Por lo tanto, la presente investigación tiene el objetivo de analizar el estigma en estudiantes de psicología, con la hipótesis de que los conocimientos y actitudes tienen valor predictivo sobre las conductas estigmatizantes.

\section{MÉTODO}

El presente estudio fue cuantitativo, analítico y transversal.

\section{Participantes.}

La muestra fue por conveniencia y se compuso de 864 estudiantes de la licenciatura en psicología de tres universidades públicas mexicanas, seleccionados de manera no probabilística, se incluyeron estudiantes mayores de 18 años, que aceptaron participar de forma voluntaria mediante la firma del consentimiento informado. Se excluyeron a los alumnos que tuvieron alguna dificultad motriz o cognitiva que les impidió contestar de forma autoadministrada los instrumentos de medición, y aquellas encuestas que no fueron respondidas en su totalidad. 


\section{Instrumentos.}

Encuesta sociodemográfica, que incluía datos sobre sexo, edad, universidad y semestre de los participantes. En este cuestionario también se incluyó una pregunta que exploraba el número de materias relacionadas con la salud y EM que habían cursado durante su trayectoria escolar.

Las escalas de medición utilizadas fueron adaptadas y validadas previamente por los autores de este estudio (aún sin publicar) traducidas al español mediante el procedimiento de traducción-retraducción, corroborando la equivalencia semántica de los ítems a través de un grupo focal, y la versión final de los reactivos en español fue acordada por el equipo de investigación.

La primera escala utilizada fue la Escala de Conocimiento de la Salud Mental. Mental Health Knowledge Schedule (MAKS), (Evans-Lacko et al., 2010). Un cuestionario autoadministrado que evalúa conocimientos sobre la EM a través de 12 reactivos, los primeros 6 son afirmaciones que exploran el nivel de conocimiento sobre salud mental relacionadas con el estigma (búsqueda de ayuda, reconocimiento, apoyo, empleo, tratamiento y recuperación) y los últimos 6 reactivos exploran el grado en el que el participante ubica alguna de las siguientes condiciones como una EM: depresión, estrés, esquizofrenia, trastorno bipolar, drogadicción, duelo. Se responde en una escala tipo Likert con 5 opciones de respuesta, evaluadas de 1 a 5 , donde 5 es totalmente de acuerdo y 1 totalmente en desacuerdo; "no sé" se codifica como neutral (3), de forma que, a mayor puntuación, mayor conocimiento. Tal como afirman los autores del estudio original, el Análisis Factorial Exploratorio realizado con la muestra de este estudio confirmó que MAKS no es unidimensional por la presencia de diferentes dominios que no se correlacionan entre sí, el manuscrito con las propiedades de este 
instrumento no ha sido publicado en muestra de estudiantes mexicanos. Por lo cual, para su análisis se consideran solamente las puntuaciones de cada reactivo.

La segunda escala empleada fue la Escala de Actitudes. Clinician's Attitudes (MICA-4), (Kassam, Glozier, Leese, Henderson y Thornicroft, 2010). La versión 4 se trata de una escala validada para el personal y los estudiantes de una amplia gama de profesiones de la salud. El objetivo de la escala consiste en mostrar el tipo de actitudes (positivas o negativas) predominantes en estudiantes de la salud hacia las personas con problemas de salud mental. El cuestionario autoadministrado original cuenta con 16 reactivos en una escala unidimensional con respuestas en formato Likert que se puntúan de 1 a 6 , donde 1 es totalmente de acuerdo y 6 totalmente en desacuerdo; a mayor puntaje se considera una actitud más negativa. La validación a través del Análisis Factorial Exploratorio (AFE) en estudiantes mexicanos del área de ciencias de la salud, llevada a cabo con la muestra de este estudio y cuyo manuscrito al respecto no ha sido publicado, llevó a eliminar 11 de los 16 reactivos originales quedando una escala conformada de 5 reactivos distribuidos en dos factores. El factor 1 fue nombrado "trato denigrante" $(\alpha=.54)$, constituido por 3 reactivos que incluyen afirmaciones como: "Ser un profesional en el área de la salud mental no es ser un verdadero profesional de la salud", "Si un psiquiatra me diera instrucciones para tratar a las personas con una enfermedad mental de manera irrespetuosa seguiría sus instrucciones" y "Usaría los términos "loco", "desquiciado", "demente", etc. para describir a las personas con enfermedades mentales que he conocido". El factor 2 se denominó "temor al rechazo" ( $\alpha=.76)$ e incluye 2 reactivos: "Si tuviera una EM me daría vergüenza comunicarlo a mis amigos por temor a ser tratado diferente" y "Si tuviera una EM nunca lo admitiría ante mis colegas por temor a ser tratado diferente" 
También se aplicó la Escala de Comportamiento Informado e Intencional. Reported and Intended Behaviour Scale (RIBS), (Evans-Lacko et al., 2006). Se trata de una escala de 8 reactivos. Los primeros 4 reactivos evalúan experiencias previas de convivencia con personas con alguna EM y se responden con las opciones "si", "no" y "no sé", a esta parte del cuestionario se le denominó (RIBS2). Los últimos 4 reactivos evalúan la disposición a vivir, trabajar, estar cerca o tener amistad con alguna persona que padezca una EM, esto se hace en una escala Likert que va del 1 (totalmente en desacuerdo) a 5 (totalmente de acuerdo), de forma que, a mayores puntuaciones, se refleja una mayor disposición del comportamiento para convivir con personas con alguna EM. Para efectos del análisis de la conducta intencionada de cada participante se considera la suma de estos últimos 4 reactivos, la cual según los autores de la escala y nuestro propio análisis con la muestra de este estudio tuvo una estructura unifactorial con $\alpha=.86$ (resultados no publicados).

\section{Procedimiento.}

El proyecto de investigación contó con la aprobación del Comité de Ética en Investigación del Instituto de Investigaciones Psicológicas de la Universidad Veracruzana, el cual cuenta con registro en la Comisión Nacional de Bioética (CONBIOÉTICA), así como con el permiso de las autoridades universitarias de las instituciones donde se llevó a cabo la recolección de datos.

Para la aplicación de los instrumentos, se acudió a las aulas y espacios libres de las distintas universidades, en donde se abordó a los posibles participantes y se les informó del objetivo de la investigación, haciendo énfasis en que se trataría de una participación voluntaria no remunerada, en la cual sus datos serían estrictamente confidenciales. Quienes aceptaron 
participar firmaron un consentimiento informado y respondieron los cuestionarios en un tiempo aproximado de 10 minutos. La aplicación se llevó a cabo en los meses de octubre y noviembre del 2019.

\section{Análisis de datos.}

Los datos sociodemográficos se analizaron a través de estadística descriptiva con medias y frecuencias. Para el análisis inferencial se emplearon técnicas no paramétricas debido a la no normalidad en la distribución de los datos, misma que se comprobó a través de la prueba de Shapiro-Wilk.

Para iniciar el análisis inferencial se dicotomizaron en dos grupos los puntajes de la variable dependiente "conductas de discriminación" utilizando la sumatoria total de cada individuo reportada en los primeros 4 reactivos de la escala RIBS. El primer grupo fue denominado "discrimina", y estuvo conformado por participantes que tenían entre 4 y 15 puntos totales y el segundo "no discrimina" tenía puntuaciones mayores a 15.

Una vez realizada la dicotomización, se analizaron las diferencias entre los rangos medios para las variables continuas de las variables sociodemográficas y psicológicas entre ambos grupos por medio de la prueba U de Mann Whitney, así como los puntajes de las 4 primeras preguntas del RIBS por medio de la prueba de $\mathrm{x}^{2}$. Posteriormente se llevó a cabo un análisis de regresión logística binaria para analizar relaciones causales entre la variable dependiente "conductas de discriminación" y las variables independientes: conocimiento sobre salud mental y actitudes hacia los pacientes psiquiátricos. Todos los datos se analizaron con el programa SPSS versión 20 y el alfa prefijado fue de 95. 


\section{RESULTADOS}

\section{Características sociodemográficas de los participantes.}

La muestra final del estudio estuvo conformada por 864 estudiantes: 207 del primer semestre, 163 del tercer semestre, 165 del quinto semestre, 175 del séptimo semestre, y 154 del noveno. La edad promedio fue de 21.4 años \pm 4.2 DS y el $72.5 \%$ de las participantes $(n=626)$ fueron mujeres. La frecuencia de participantes por universidad fue: 324 estudiantes $(37.5 \%)$ de la Universidad Autónoma del Estado de México, 295 de la Universidad Veracruzana (34.1\%) y 245 de la Universidad Autónoma de Ciudad Juárez (28.4\%). Cabe mencionar que la mediana de número de materias cursadas relacionadas con la enfermedad mental fue 1 .

\section{Comparación entre los grupos "discrimina" y "no discrimina".}

En función del semestre y la edad existieron diferencias significativas entre los grupos (ver tabla 1), de tal forma que, a mayor edad y mayor grado de estudios, existe mayor disposición a convivir con personas con alguna enfermedad mental. También, los resultados mostraron que el grupo "no discrimina" tuvo frecuencias superiores en la de convivencias previas con personas con alguna EM (RIBS2). 


\section{Tabla 1}

Comparación entre grupos de las variables edad, semestre y antecedentes de convivencia con algún paciente psiquiátrico

\begin{tabular}{|c|c|c|c|c|c|}
\hline Variable & & $\begin{array}{l}\text { Discrimina } \\
\mathrm{n}=282\end{array}$ & $\begin{array}{l}\text { No } \\
\text { discrimina } \\
\mathrm{n}=582\end{array}$ & Sig. & * \\
\hline Semestre & & 397.96 & 449.24 & .004 & 72321.5 \\
\hline Edad & & 400.84 & 447.84 & .009 & 73134.0 \\
\hline $\begin{array}{l}\text { ¿Tienes o has tenido un amigo cercano } \\
\text { que padezca un problema de salud } \\
\text { mental? }\end{array}$ & $\begin{array}{l}\mathrm{Si} \\
\text { No } \\
\text { No sé }\end{array}$ & $\begin{array}{l}227 \\
447 \\
117\end{array}$ & $\begin{array}{l}828 \\
593 \\
219\end{array}$ & 001 & 71.188 \\
\hline $\begin{array}{l}\text { ¿Tienes o has tenido un vecino que } \\
\text { padezca un problema de salud mental? }\end{array}$ & $\begin{array}{l}\text { Si } \\
\text { No } \\
\text { No sé }\end{array}$ & $\begin{array}{l}276 \\
441 \\
134\end{array}$ & $\begin{array}{l}672 \\
652 \\
316\end{array}$ & 001 & 33.180 \\
\hline $\begin{array}{l}\text { ¿Trabajas o has trabajado con alguien que } \\
\text { padezca un problema de salud mental? }\end{array}$ & $\begin{array}{l}\text { Sí } \\
\text { No } \\
\text { No sé }\end{array}$ & $\begin{array}{l}182 \\
576 \\
93\end{array}$ & $\begin{array}{l}498 \\
905 \\
237\end{array}$ & 001 & 36.526 \\
\hline $\begin{array}{l}\text { ¿Vives o has vivido con alguien que } \\
\text { padezca un problema de salud mental? }\end{array}$ & $\begin{array}{l}\text { Si } \\
\text { No } \\
\text { No sé }\end{array}$ & $\begin{array}{l}173 \\
553 \\
125\end{array}$ & $\begin{array}{l}525 \\
871 \\
244\end{array}$ & 001 & 41.121 \\
\hline
\end{tabular}

* Valor U de Mann-Whitney o $\mathrm{X}^{2}$. La tabla fue elaborada por los autores.

Por otro lado, al realizar comparaciones entre los reactivos del MAKS y las subescalas del MICA en función del grupo "discrimina" y no discrimina", se encontraron diferencias significativas intergrupales, de tal forma que los integrantes del grupo "no discrimina" tenían mayores conocimientos y menos actitudes desfavorables. Los resultados se muestran en la tabla 2 .

Por otro lado, al realizar comparaciones entre los reactivos del MAKS y las subescalas del MICA en función del grupo "discrimina" y no discrimina", se encontraron diferencias significativas intergrupales, de tal forma que los integrantes del grupo "no discrimina" tenían mayores conocimientos y menos actitudes desfavorables. Los resultados se muestran en la tabla 2. 


\section{Tabla 2}

Comparación de rangos medios de conocimiento (MAKS) y actitudes (MICA)

\begin{tabular}{|c|c|c|c|c|}
\hline Variable & $\begin{array}{l}\text { RIBS } \\
\text { Discrimina } \\
\mathrm{n}=282\end{array}$ & $\begin{array}{l}\text { RIBS } \\
\text { No discrimina } \\
\mathrm{n}=582\end{array}$ & Sig. & $\mathrm{U}$ \\
\hline $\begin{array}{l}\text { MAKS1 La mayoría de las personas con } \\
\text { problemas de salud mental quieren tener un } \\
\text { empleo }\end{array}$ & 373.23 & 461.22 & .001 & 65349.0 \\
\hline $\begin{array}{l}\text { MAKS2 Si un amigo tiene un problema de } \\
\text { salud mental sé cómo guiarlo para que pueda } \\
\text { obtener ayuda profesional }\end{array}$ & 375.04 & 460.34 & .001 & 65858.0 \\
\hline $\begin{array}{l}\text { MAKS4 La psicoterapia puede ayudar a las } \\
\text { personas con problemas de salud mental }\end{array}$ & 395.20 & 450.57 & .001 & 71543.5 \\
\hline $\begin{array}{l}\text { MAKS7 Cree que la depresión en un tipo de } \\
\text { enfermedad mental }\end{array}$ & 375.92 & 459.92 & .001 & 66106.0 \\
\hline $\begin{array}{l}\text { MAKS8 Cree que el estrés es un tipo de } \\
\text { enfermedad mental }\end{array}$ & 476.90 & 410.98 & .001 & 69540.0 \\
\hline $\begin{array}{l}\text { MAKS9 Cree que la esquizofrenia es un tipo } \\
\text { de enfermedad mental }\end{array}$ & 405.56 & 445.55 & .008 & 74466.0 \\
\hline $\begin{array}{l}\text { MAKS10 Cree que el trastorno bipolar es un } \\
\text { tipo de enfermedad mental }\end{array}$ & 389.49 & 453.34 & .001 & 69933.5 \\
\hline $\begin{array}{l}\text { MAKS11 Cree que la drogadicción es un } \\
\text { tipo de enfermedad mental }\end{array}$ & 400.94 & 447.79 & .008 & 73161.5 \\
\hline $\begin{array}{l}\text { MICA } \\
\text { Factor 1: temor al rechazo }\end{array}$ & 479.46 & 409.74 & .001 & 68818.5 \\
\hline $\begin{array}{l}\text { MICA } \\
\text { Factor 2: trato denigrante }\end{array}$ & 481.52 & 408.75 & .001 & 68238.5 \\
\hline
\end{tabular}

Nota: Prueba U de Mann-Whitney. La tabla fue elaborada por los autores.

\section{Modelo de regresión.}

Se realizó un análisis de regresión logística binaria para comprobar si la variable dependiente (conducta de discriminación intencionada), podía ser predicha por las variables independientes: conocimiento sobre salud mental, actitudes y antecedentes de convivencia con un paciente psiquiátrico. Se eligió la regresión logística binaria debido a que la variable dependiente es una variable con dos categorías: "discrimina" y "no discrimina". Los resultados se presentan en la tabla 3. Encontramos que el modelo final de 
regresión logística es significativo $\left(\mathrm{X}^{2}=117.528, \mathrm{p}<.00\right)$, y explica $17.7 \%$ de la varianza de la variable dependiente $\left(\mathrm{R}^{2}\right.$ de Nagelkerke). La matriz de clasificación tiene un porcentaje de acierto de $70.9 \%$ de los casos, y la prueba de bondad de ajuste de Hosmer \& Lemeshow muestra un buen ajuste de los datos al modelo $(\mathrm{p}=.528)$.

\section{Tabla 3}

Modelo de regresión logística binaria, variable dependiente dicotomizada: "discrimina" y "no discrimina"

\begin{tabular}{|c|c|c|c|c|c|c|}
\hline Variables en la ecuación & $\mathrm{B}$ & S.E. & Wald & df & Sig. & $\operatorname{Exp}(B)$ \\
\hline Intercepto & -2.917 & .657 & 19.712 & 1 & .000 & .054 \\
\hline \multicolumn{7}{|l|}{ Conocimiento } \\
\hline $\begin{array}{l}\text { MAKS1 "La mayoría de las } \\
\text { personas con problemas de } \\
\text { salud mental quieren tener un } \\
\text { empleo" }\end{array}$ & .512 & .099 & 26.520 & 1 & .000 & 1.669 \\
\hline $\begin{array}{l}\text { MAKS2 "Si un amigo tiene un } \\
\text { problema de salud mental sé } \\
\text { cómo guiarlo para que pueda } \\
\text { obtener ayuda profesional" }\end{array}$ & .301 & .087 & 11.824 & 1 & .001 & 1.351 \\
\hline $\begin{array}{l}\text { MAKS10 "Cree que el } \\
\text { trastorno bipolar es un tipo de } \\
\text { enfermedad mental" }\end{array}$ & .331 & .090 & 13.432 & 1 & .001 & 1.392 \\
\hline \multicolumn{7}{|l|}{ Actitudes } \\
\hline MICA1 "temor al rechazo" & -.118 & .034 & 11.963 & 1 & .001 & .889 \\
\hline MICA2 "trato denigrante" & -.072 & .030 & 5.573 & 1 & .018 & .931 \\
\hline \multicolumn{7}{|l|}{ Antecedentes de convivencia } \\
\hline $\begin{array}{l}\text { "¿Has tenido un vecino que } \\
\text { padezca un problema de salud } \\
\text { mental?" }\end{array}$ & .331 & .090 & 13.545 & 1 & .000 & 1.392 \\
\hline $\begin{array}{l}\text { "¿Tienes o has tenido un amigo } \\
\text { cercano que padezca un } \\
\text { problema de salud mental?" }\end{array}$ & .227 & .092 & 6.090 & 1 & .014 & 1.254 \\
\hline
\end{tabular}




\section{DISCUSIÓN}

\section{Edad y discriminación.}

Loshallazgos de este estudio muestran que los participantes que conformaban el grupo denominado "no discrimina" tuvieron significativamente mayor edad promedio que el grupo "discrimina", lo cual coincide con lo reportado en otros estudios realizados con muestras de enfermeras psiquiátricas chinas (Hsiao, Lu y Tsai; 2015), población general inglesa (Evans-Lacko, Corker, Williams, Herderson y Thornicroft, 2014), y población general mexicana (García, 2017). Por el contrario, en un estudio reciente de Natera y Casco (2014) llevado a cabo en población general y profesionales de la salud mexicanos, se reportó que las personas de mayor edad discriminaban más a personas con alguna enfermedad mental; lo mismo se encontró en un estudio con población general eslovaca (Letovancová y Kovalcikova, 2017). Como se puede apreciar, la asociación entre edad y discriminación ha sido reportada en diversos estudios realizados en diferentes muestras poblacionales, aunque todavía quedan pendientes trabajos que exploren los aspectos psicosociales que podrían estar influyendo en la formación de actitudes y conductas hacia la EM en diversas generaciones.

\section{Años de formación universitaria y actitudes hacia la EM.}

Se encontró que los alumnos del grupo "no discrimina" se encontraban en un curso escolar más avanzado que los sujetos del grupo "discrimina", como también fue reportado por Medina-Mesa et al. (2019) en estudiantes de ciencias de la salud españoles y Ay et al. (2006) en estudiantes de medicina turcos. En el mismo sentido, Brucker y Doty (2019) encontraron que el personal clínico y administrativo de las agencias de salud mental en Estados Unidos con niveles de educación más altos tenían una actitud más favorable que aquellos con menos educación, lo mismo ocurrió en un 
estudio de García (2017) en población general mexicana. Considerando lo anterior, se sugiere que, a mayor formación académica se incrementan las posibilidades de desarrollar actitudes y conocimientos asociados a la disminución de la estigmatización de los pacientes psiquiátricos. En México los programas de licenciatura en el área de ciencias de la salud, incluyen materias de psiquiatría y psicopatología en semestres avanzados, este hecho también podría estar relacionado con este hallazgo.

\section{Conocimientos y conductas de discriminación.}

Los resultados muestran que los estudiantes de psicología que conformaban el grupo "no discrimina" tuvieron mayores conocimientos generales respecto a la EM que los del grupo “discrimina”. La asociación entre este tipo de conocimientos y la discriminación hacia pacientes psiquiátricos, ha sido previamente reportada en otros estudios realizados en muestras de profesionales de la salud en países como la India (Gandhi et al. 2019), España (Sanz, 2018) y Portugal (Fernandes, Santos, Moreira, Vargas y Nóbrega, 2019), así como en población general española (Moreno, Sánchez, y López, 2014); en investigaciones realizadas con estudiantes españoles de secundaria (Rodríguez-Meirinhos y Antolín-Suárez, 2020) y universitarios ingleses (Simmons, Jones, y Bradley, 2017). Incluso este hallazgo se reproduce según lo reportado en una revisión sistemática de estudios llevados a cabo en muestras de población general de diferentes países (Choudhry, Mani, Ming y Khan, 2016).

En cuanto a los conocimientos sobre enfermedades mentales específicas, en este estudio se encontró que quienes identificaron al trastorno bipolar (TB) como una enfermedad mental, tuvieron menos probabilidades de expresar conductas de discriminación hacia pacientes psiquiátricos, lo que se corresponde con lo mencionado por Fresán, Berlanga, Robles- 
García, Álvarez-Icaza y Vargas-Huicochea (2013), quienes afirman que la alfabetización en salud mental en estudiantes universitarios de la salud mexicanos, dirigida a mejorar el conocimiento de las características del $\mathrm{TB}$, constituye un elemento esencial para reducir el estigma. Nuestro hallazgo adquiere mayor relevancia si se toma en cuenta lo referido por Vargas-Huicochea et al. (2017), quienes encontraron que sólo el 3.8\% de estudiantes de medicina mexicanos reconocieron los síntomas y el tratamiento más adecuado del TB. Además, a nivel mundial hasta el $40 \%$ de los pacientes con TB son mal diagnosticados (Hirschfeld y Vornik, 2003). Esto debe ser tomado en cuenta por profesores y autoridades educativas de escuelas de psicología y otras ciencias de la salud, con el fin de incluir una formación sólida sobre las características específicas de cada trastorno mental.

Por otra parte, los resultados de este estudio muestran que saber guiar a un amigo en la búsqueda de atención psiquiátrica, predice conductas de menos discriminación, lo que coincide con lo encontrado por Davies, Beever y Glazebrook (2018), quienes reportaron que mejores actitudes hacia la EM se asocia con mayores probabilidades de que ayuden a un amigo cercano a encontrar ayuda psiquiátrica en caso necesario. Por lo que, incluir protocolos de atención psiquiátrica al currículo de estudiantes de psicología, podría mejorar sus actitudes y conductas hacia quienes padecen una EM.

Además, en nuestra investigación se encontró que el hecho de considerar que una persona con problemas de salud mental quiere tener un empleo, predice conductas menos discriminatorias hacia este grupo de pacientes. Reconocer las necesidades psicosociales (como la obtención de un empleo) de quienes padecen un diagnóstico psiquiátrico, es un elemento clave para 
tener mejores actitudes hacia la EM. Al respecto, se ha encontrado en diversos estudios que algunos pacientes psiquiátricos son rechazados por los empleadores cuando estos se enteran de sus padecimientos, pues los consideran poco saludables para sus organizaciones (Brouwer, 2020). Esto se traduce en menores oportunidades y experiencia laboral para este grupo de pacientes (Charette-Dussault y Corbiére, 2019). Conviene entonces enfatizar en la formación de estudiantes de psicología que, al igual que todo individuo, las personas con alguna EM pueden llegar a ser capaces de desarrollarse laboralmente y que la obtención de un empleo remunerado podría darles la satisfacción de tener seguridad financiera, socializar, y tener un sentido de vida.

\section{El temor al rechazo.}

Nuestros hallazgos indican que temer al rechazo en caso de padecer una EM, predice conductas de discriminación. Diversos estudios indican que muchos pacientes psiquiátricos prefieren ocultar sus diagnósticos a sus pares por temor a ser estigmatizados y tener menores oportunidades de un ascenso laboral (Brouwer, 2020; Brohan et al., 2012), este resultado se ha replicado en muestras de estudiantes de la salud (Chad et al., 2017) y personal que labora en medios hospitalarios (Barber, 2019; Waugh, Lethem, Shering y Henderson, 2017).

Conviene mencionar que, en el caso de los resultados de este estudio, justamente ese temor al rechazo en caso de padecer una EM, explica las conductas de discriminación hacia quienes padecen un diagnóstico psiquiátrico $\mathrm{y}$, como se señaló anteriormente, se trata de un temor que comparten personas alrededor del mundo; incluso quienes laboran en el área de la salud. A pesar de los múltiples esfuerzos por sensibilizar a las poblaciones respecto a la inclusión social y laboral de personas con este 
tipo de condiciones de salud mental, el estigma social hacia ellos continúa, y parece interiorizarse en cada sujeto en forma de miedo al rechazo en caso de tener un diagnóstico psiquiátrico. En este sentido, conviene resaltar la importancia de realizar acciones educativas que promuevan el cambio social, de tal forma que se promueva la visualización de la EM como una condición de salud que no debería acompañarse de segregación social y laboral.

\section{Psiquiatría y trato denigrante.}

Como resultado relevante se encontró que, cuando existe una mayor tendencia a considerar a la psiquiatría como una verdadera profesión incluida en el área de la salud, y a tratar con respeto a un paciente psiquiátrico, esto actúa como predictor de conductas menos discriminatorias. Este hallazgo es de gran importancia si se toma en cuenta que la psiquiatría es una rama de la medicina poco valorada (Zhu et al., 2018), y que, además, muchos profesionales de la salud utilizan términos como "loco" para estigmatizar a los pacientes psiquiátricos (Ozer, Varlik, Ceri, Ince y Arslan, 2017). Incluso, se ha documentado que trabajadores de la psiquiatría tienen actitudes desfavorables y estigmatizantes hacia los pacientes, lo cual se ve expresado en el uso de la reclusión y la restricción física innecesaria como medio de control (Giacchero et al., 2017; Cowman, Björkdahl, Clarke, Gethin y Maguire, 2017). Esto genera percepciones de un trato coercitivo, humillante y opresivo en los receptores de los servicios de salud mental (Nyttingnes, Ruud y Rugkasa, 2017).

Gran parte de las actitudes hacia la EM y hacia los profesionales involucrados en su tratamiento pueden ser formadas o modificadas en el contexto universitario. Es por ello que estos hallazgos deberían ser contemplados en el proceso de toma de decisiones para la planeación 
curricular de las escuelas de psicología, tomando en cuenta que los futuros profesionales del comportamiento humano formarán parte de equipos de trabajo encargados de la prevención y el tratamiento a personas con diagnósticos psiquiátricos.

\section{Convivir con la enfermedad mental.}

Según los resultados del presente trabajo, los estudiantes que no discriminan reportaron una mayor convivencia con personas que padecen alguna EM en su entorno social cercano, incluso este tipo de experiencias con un amigo o vecino, son predictoras de comportamientos menos estigmatizantes.

La asociación entre convivir con un paciente psiquiátrico y tener comportamientos de aceptación hacia ellos fue también reportada en investigaciones con muestras de estudiantes del área de la salud en diferentes partes del mundo (Medina, Martínez, Morales y Gill, 2018; Papish et al. 2013; Sastre, 2019; Lyndon, Crowe, Wuensch, McCammon y Davis, 2016; Parra, Ortíz y Moya, 2017; Sawsan, Reem, Kimberly y Muayyad, 2019; Tomas, 2020; Eksteen, Becker y Lippi, 2017; Zhu et al., 2018; Aflakseir, Rasoolo, Goosarzi y Molazadeh, 2019), y en población general española según Frías et al. (2019). Por lo tanto, existe sólida evidencia para considerar que el contacto personal, familiar y el laboral con personas que padecen alguna EM, predice conductas de menos rechazo hacia los pacientes psiquiátricos. El contacto frecuente y las relaciones familiares o afectivas que se podrían establecer con este tipo de pacientes, constituyen una valiosa oportunidad para erradicar los prejuicios y desarrollar pautas de interacción basadas en el respeto y la aceptación (Grandón, Saldivia, Cova, Bustos y Turra, 2018). 


\section{Limitaciones}

Los resultados aquí reportados se deben interpretar considerando las limitaciones del estudio. En principio, el hecho de que se llevó a cabo con una muestra por conveniencia no permite la generalización de los resultados. Por otro lado, la aplicación de los cuestionarios se realizó en el contexto universitario, lo que pudo tener influencia en las respuestas a modo de deseabilidad social. Además, al utilizar muestras de diferentes regiones mexicanas y considerando las diferencias culturales, los resultados de futuras investigaciones que aborden el estigma hacia los pacientes psiquiátricos en universitarios, podrían enriquecerse si se incluyen algunos datos cualitativos que permitan un conocimiento más profundo de las concepciones subjetivas de los estudiantes respecto a la EM.

Es recomendable que otros estudios sobre esta temática sean realizados con escalas diseñadas ex profeso para la cultura latinoamericana, puesto que es limitada todavía la existencia de instrumentos que reflejen la idiosincrasia local sobre el asunto de la discriminación; hacerlo podría mejorar la confiabilidad de los datos que se obtengan.

\section{Conclusiones}

Los resultados de este estudio sugieren que los estudiantes de psicología de semestres superiores y con mayor edad tienen mejores actitudes hacia la EM. Además, el conocimiento, las actitudes y la experiencia de convivir con algún paciente psiquiátrico son predictores de conductas de menos discriminación. Estos hallazgos brindan sólida evidencia de la necesidad de generar estrategias que promuevan en los estudiantes de psicología el conocimiento sobre la EM y la convivencia cercana con pacientes psiquiátricos con el fin de disminuir el estigma hacia estos. 


\section{REFERENCIAS}

I Ahmedani B. K. (2011). Mental Health Stigma: Society, Individuals, and the Profession. Journal of social work values and ethics, 8(2), 41-416. Recuperado de https:/www.ncbi.nlm.nih.gov/pmc/articles/ PMC3248273/

I Aflakseir, A., Rasoolo, M., Goosarzi, M., y Molazadeh, J. (2019). Personas con enfermedad mental y reducción del estigma: un estudio transversal en un grupo de estudiantes universitarios. Irán Journal de Psiquiatria, 14(4), 297-301. Recuperado de https://www.ncbi.nlm.nih.gov/pmc/articles/PMC7007504/

I Alonso, R., Lorenzo, L., Flores, I., Martín, J., y García, L. (2019). El psicólogo clínico en los centros de salud. Un trabajo conjunto entre atención primaria y salud mental. Atención Primaria, 51(5), 310-313. Recuperado de https://www.ncbi. nlm.nih.gov/pmc/articles/PMC6836879/

I Ay, P., Save, D., y Fidanoglu, O. (2006). ¿El estigma relacionado con los trastornos mentales difiere a través de la educación médica? Social Psychiatry y Psychiatry Epidemiol, 41, 63-67. Doi: 10.1007/s00127005-0994-y

I Bannatyne, A., J., y Stapleton, P. B. (2016). Actitudes hacia la anorexia nerviosa: diferencias de estigma volitivo en una muestra de estudiantes de medicina y psicología preclínicos. Revista de salud mental, 26(5), 442-448. Recuperado de https://doi.org/10.3109/09638237.2016.1149801

I Barber, Ch. (2019). Profesionales de la salud que experimentan problemas de salud mental. Revista británica de enfermería de salud mental, 8(2). Recuperado de https://doi.org/10.12968/bjmh.2019.0005

I Brohan, E., Henderson, C., Trigo, K., Malcolm, E., Clement, S., Cebada, A. Slade, M., y Thornicroft, G. (2012). Revisión sistemática de creencias, comportamiento y factores de influencia asociado con la divulgación de un problema de salud mental en el lugar de trabajo. Psiquiatría BMC, 12(11), 1-7. Recuperado de https://doi.org/10.1186/1471-244X-12-11

$\checkmark$ Brouwer, E. (2020). El estigma social es un factor que contribuye al desempleo en personas con enfermedades mentales o problemas de salud mental: investigación de posición y direcciones futuras. BMC Psychology, 8(36), 1-7. Recuperado de https://doi.org/10.1186/s40359-020-00399-0

I Brucker, D., y Doty, M. (2019). Actitudes del personal de los centros comunitarios de salud mental sobre el empleo de personas con enfermedades mentales graves. Revista de rehabilitación psiquiátrica, 42(1), 32-40. Recuperado de https://psycnet.apa.org/buy/2018-49537-001

I Cowman, S., Björkdahl, A., Clarke, E., Gethin, G., y Maguire, J. (2017). Un estudio de encuesta descriptiva sobre la gestión de la violencia y las prioridades entre el personal psiquiátrico de los servicios de salud mental, en diecisiete países europeos. BMC Servicios de salud, 17(59), 1-7. doi: org/10.1186/s12913-0171988-7

I Chin, S. H., \& Balon R. (2006). Attitudes and perceptions toward depression and schizophrenia among residents in different medical specialties. Academic Psychiatry, 30(3), 262-263. doi:10.1007/s10597-0109341-1

I Chand, S., Lin, H., Seow, E., Yiang, B., Abdin, E., Samari, E., Lin, W., Chong, S., y Subramaniam, S. (2017) Estigma hacia la enfermedad mental entre estudiantes de medicina y enfermería en Singapur: un estudio transversal. BMJ Open, 7(12), 1-11. doi:10.1136/bmjopen-2017-018099 
I Charette-Dussault, E., y Corbiére, M. (2019). Una revisión integral de las barreras a la adquisición de empleo para personas con enfermedades mentales graves. The Journal of nervous and mental disease, 207(7), 523-537. Recuperado de https://journals.lww.com/jonmd/Abstract/2019/07000/An_Integrative_ Review_of_the_Barriers_to_Job.1.aspx

I Choudhry, F., Mani, V., Ming, L., y Khan, T. (2016). Creencias y percepciones sobre problemas de salud mental: una meta-síntesis. Neuropsychiatric disease and treatment, 12, 2807-2818. Recuperado de https:// www.ncbi.nlm.nih.gov/ pmc/articles/PMC5096745/

I Davies, E., Beever, E., y Glazebrook, C. (2018). Un estudio piloto controlado aleatorio del curso de aprendizaje en línea sobre primeros auxilios en salud mental con estudiantes de medicina del Reino Unido. BMC Medical education, 18(45), 1-12. doi: 101186/s12909-018-1154-x.

I Eksteen, H-C., Becker, P., y Lippi, G. (2017). Estigmatización hacia los enfermos mentales: percepciones de psiquiatras, estudiantes de medicina de rotación preclínica y pos clínica. Revista internacional de psiquiatría social, 63(8), 782-791. doi: 10.1177 / 0020764017735865

I Evans-Lacko, S., Rose,D., Little, K., Flach, C., Rhydderch,D., Henderson, C., y Thornicroft, G. (2006). Development and psychometric properties of the Reported and intended behaviour scale (RIBS): a stigma-related behaviour measure. Epidemiology and Psychiatric Sciences, 20(3), 263-271. doi: 10.1017/ S2045796011000308

I Evans-Lacko, S., Little, K., Meltzer H.,Rose, D., Rhydderch, D., Henderson, C., y Thornicroft, G. (2010). Development and Psychometric Properties of the Mental Health Knowledge Schedule. La Revue canadienne de psychiatrie, 55(7), 440-448. doi.org/10.1177\%2F070674371005500707

Ittps://journals.sagepub.com/doi/abs/10.1177/00 20764017735865

I Evans-Lacko, S., Corker, E., Williams, P., Herderson, C., y Thornicroft (2014). Efecto de la campaña contra el stigma Time to Chance" sobre las tendencias del estigma público relacionado con las enfermedades mentales entre la población inglesa en 2003-2013: un análisis de datos de encuestas. The Lancet Psychiatry, 1(2), 121-128. Recuperado de https://outlook.live.com/mail/inbox

I Fernandes, C., Santos, W., Moreira, W., Vargas, D., y Nóbrega, M. (2019). Opinions on mental illness from the perspective of primary care nurses in Portugal, Revista Gaúcha de Enfermagem, 40, 1-8. Recuperado de https://www.scielo. br/pdf/rgenf/ v40/1983-1447-rgenf-40-e20190034.pdf

I Fresán, A., Robles, R., Cota, M., Berlanga, C., Lozano, D., y Tena, A. (2012). Actitudes de mujeres estudiantes de psicología hacia las personas con esquizofrenia: Relación con la percepción de agresividad y peligrosidad. Salud Mental, 35(3), 215-223. Recuperado de https://www.redalyc.org/pdf/582/5822 3340005.pdf

I Fresán, A., Berlanga, C., Robles-García, R., Álvarez-Icaza, D., Vargas-Huicochea, I. (2013). Alfabetización en salud mental en el trastorno bipolar: asociación con la percepción de agresividad y el género en estudiantes de medicina. Salud mental, 36(3). Recuperado de http://www.scielo.org.mx/scielo. php?pid=S0185 33252013000300008\&script=sci_arttext 
I Frías, V., Fortuny, J., Guzmán, S., Santamaría, P., Martínez, M., y Pérez, V. (2018). Estigma: la relevancia del contacto social en el trastorno mental. Enfermería Clínica, 28(2), 111-117. Recuperado de https://www. sciencedirect.com/science/article/abs/pii/S1130862117300803

I Gandhi, S., Poreddi, V., Govindan, R., Jothimani, G., Anjanappa, S., Sahu, M., Narayanasamy, P., Manjunath, N., Naveenkumar, C., y Badamath, S. (2019). Conocimientos y percepciones de las enfermeras de atención primaria hindúes sobre las enfermedades mentales. Investigación y Educación en enfermería, 37(1). Recuperado de https://pesquisa.bvsalud.org/portal/resource/es/mdl-31083843

I García. S. (2017) Las actitudes hacia la enfermedad mental y el psiquiatra: resultados preliminares. Acta psiquiátrica y psicológica de América Latina, 39(2), 159-168. Recuperado de http://repositorio.inprf.gob. $\mathrm{mx} /$ handle/12345 6789/4844?show=full

I Giacchero, K., Silva, D., Arena, C., Ciccone, B., Guidorizzi, A., Inocenti, A., y Longo, T. (2017). Contención física y mecánica en unidades psiquiátricas: percepciones y experiencias del personal de enfermería. Archivos de enfermería psiquiátrica, 32(3), 367-372. Recuperado de https://doi.org/10. 1016/j. apnu.2017.11.027

$\checkmark$ Gilaberte, I., Failde, I., Salazar, A., \& Caballero, L. (2012). Actitud de los alumnos de quinto de medicina hacia la salud mental. Educación Médica, 15(4), 227-233. Recuperado de http://scielo.isciii.es/pdf/edu/ v15n4/original3.pdf

I Grandón,P., Saldivia, S., Cova, F., Bustos, C., y Turra, V. (2016). Análisis psicométrico y adaptación de la escala de actitudes comunitarias hacia la enfermedad mental (CAMI) en una muestra chilena. Universitas Psychologica, 15(2), 153-162. doi.org/10.11144/Javeriana.upsy15-2.apae

I Hasan, M., y Thornicroft, G. (2018). Campañas en los medios de comunicación para reducir el estigma de salud mental en Bangladesh. Lancet Psychiatry, 5(8), 616. doi: 10.1016/S2215-0366(18)30219-0.

I Hirschfeld R., y Vornik L. (2003). Reconocimiento y diagnóstico del trastorno bipolar. The Jounal of Clinical Psychiatry, 65(15), 5-9. Recuperado de https://europe pmc.org/article/med/15554789

$\checkmark$ Hsiao, C., Lu, H., y Tsai, Y. (2015). Factores que influyen en las actitudes de las enfermeras de salud mental hacia las personas con enfermedades mentales. International Journal of Mental Health Nursing, 24(3), 272-280. Recuperado de https://onlinelibrary.wiley.com/doi/abs/10.1111/inm.12129

I Kassam, A., Glozier, N., Leese, M., Henderson, C., y Thornicroft, G. (2010). Desarrollo y capacidad de respuesta de una escala para medir las actitudes de los médicos hacia las personas con enfermedades mentales (versión para estudiantes de medicina). Acta Psychiatr Scan, 122(2), 153-161. Recuperado de https://onlinelibrary.wiley.com/doi/abs/10.1111/j.1600-0447.2010.01562.x

I Letovancová, K., y Kovalcikova, N. (2017). Attitude of society towards people with mental illness: the result of national survey of the Slovak population. International Journal of Social Psychiatry, 63(3), 255260. Recuperado de https://journals.sagepub.com/doi/abs/10.1177/0020764017696334

I López, M., Fernández, L., Laviana, M., Aparicio, A., Perdiguero, D. y Rodríguez, A. (2010). Problemas de salud mental y actitudes sociales en la ciudad de Sevilla. Resultados generales del estudio "Salud mental: imágenes y realidades". Revista de la Asociación Española de Neuropsiquiatría, 30(106), 219248. Recuperado de http://scielo.isciii.es/scielo.php?script=sci_arttext\&pid=S0211-57352010000200003 
I Lyndon, A., Crowe, A., Wuensch, K., McCammon, S., y Davis, K. (2016). Estigmatización de los estudiantes universitarios de las personas con enfermedades mentales: familiaridad, teoría implícita de la persona y atribución. Revista de la salud mental, 28(3), 255-259. Recuperado de https://www.tandfonline. com/doi/abs/10.1080/09638237.2016.1244722

I Magliano, L., Read, J., Patalano, M., Sagliocchi, M., Oliviero, N., D’Ambrosio, A., Campitiello, F., Zaccaro, A., Guizzaro, A., \& Cerrato, F. (2012). Contrarrestar el estigma hacia las personas con esquizofrenia en el ámbito sanitario: una experiencia piloto en una muestra de estudiantes italianos de medicina. Psychology, Society, \& Education, 4(2), 169-181. Recuperado de http://repositorio.ual.es/bitstream/handle/10835/2866/ Magliano.pdf?sequence=1

I Marques, A., Barbosa, T., y Queirós, C. (2010). O Estigma na Doença Mental Perspectivado por Futuros Profissionais de Saúde Mental. En III Congreso de la Federación Española de Asociaciones de Rehabilitación Psicosocial. Congreso llevado a cabo en Valladolid, España. Recuperado de aberto.up.pt/ bitstream/10216/44483/2/15403.pdf

$\checkmark$ Meru, U. (2017). Estigma y creencias sobre la recuperación en relación con la enfermedad mental, impacto de la pasantía clínica en las actitudes de los estudiantes de psicología. (tesis de doctorado) Instituto de Administración de Empresas, Pakistan. Recuperado de https://ir.iba.edu.pk/ugrad-projects/57

$\checkmark$ Medina, M. E., Real, T., y Amador, N. (2015). Identificación y diagnóstico de los trastornos psiquiátricos. En Medina, M., Sarti, E., y Real, T. (Eds.), La enfermedad mental en México. La depresión y otros trastornos psiquiátricos (pp. 13-28). México: CONACYT. Recuperado de https://www.anmm.org.mx/ publicaciones/CAnivANM150/L27_ANM_DEPRESION.pdf

I Medina, Y., Martínez, C., Morales, L., y Gil, P. (2018). Las actitudes del alumnado universitario frente a las personas con trastorno mental. International Journal of Developmental and Educational Psychology 3(1), 209-218. Recuperado de http://www.infad.eu/RevistaINFAD/OJS/index.php/IJODAEP/article/view/1258

I Medina-Mesa, Y., Cruces-Montes, S., Merchán-Clavellino, A., Romero-Moreno, A., y Guil-Bozal, R. (2019). La influencia de la formación en las actitudes hacia las personas con trastorno mental en una muestra de estudiantes universitarios. International Journal of Developmental and educational psychology, 5(1). Recuperado de http://infad.eu/RevistaINFAD/OJS/index.php/IJODAEP/article/ view/1602

$\checkmark$ Moreno, A., Sánchez, F., y López, M. (2014). Diferencias de la población general en relación a los conocimientos, actitudes y conductas hacia el estigma en salud mental. Psychology, Society \& Education, 6(1), 17-26. Recuperado de http://ojs.ual.es/ojs/index.php/psye/article/view/505/483

I Navarro, N., y Trigueros, R. (2019). Estigma en los profesionales de la salud mental: una revisión sistemática. Psicología, sociedad y educación, 11(2), 253-266. Recuperado de https://doi10.25115/psye. v10i1.2268

I Natera, G., y Casco, M. (2014). Actitudes hacia la enfermedad mental en población general y en grupo de profesionales de la salud. Anales del Instituto Mexicano de Psiquiatría. Reseña de la VI Reunión de Investigación, 2(2), 176-182. Recuperado de http://repositorio.inprf.gob.mx/handle/123456789/6457 
I Nordt, C., Rössler, W., Lauber, C. y Schizophr, B. (2006). Attitudes of mental health professionals toward people with schizophrenia and major depression. Schizophenia Bulletin, 2006, 32(4), 709-14. doi. org $/ 10.1093 /$ schbul/sbj065

$\checkmark$ Nyttingnes, O., Ruud, T., y Rugkasa, J. (2016). Es increíble humillante: las expresiones de los pacientes sobre los efectos negativos de la coerción en la atención de salud mental. Revista internacional de derecho y psiquiatría, 49 (A), 147-153. Recuperado de https://doi.org/10.1016/j.ijlp.2016.08.009

1 Organización de las Naciones Unidad (2005). El derecho a la salud mental como una de las preocupaciones de las Naciones Unidad en ACHNU (20 de septiembre de 2020). Recuperado de http://www.unesco.org/ new/fileadmin/MULTIMEDIA/FIELD/Montevideo/pdf/SaludMentalOneUNWeb.pdf

I Organización de las Naciones Unidas (2018). Consulta sobre derechos humanos y salud mental: "Identificación de estrategias para promover los derechos humanos en la salud mental" en ACHNU (20 de septiembre de 2020). Recuperado de https://www.ohchr.org/EN/NewsEvents/Pages/DisplayNews. aspx?NewsID $=23080 \&$ LangID $=\mathrm{E}$

1 Organización de las Naciones Unidas. (20 de septiembre de 2020). Relator especial sobre el derecho de toda persona al disfrute del más alto nivel posible de salud fisica y mental. Recuperado de https://www. ohchr.org/SP/Issues/Health/Pages/ SRRight HealthIndex.aspx

I Ozer, U., Varlik, C., Ceri, V., Ince, B., y Arslan, M. (2017). El cambio comienza con nosotros: actitudes estigmatizantes hacia las enfermedades mentales y el uso de lenguaje estigmatizante entre los profesionales de la salud mental. La revista de psiquiatría y ciencias neurológicas, 3(30), 224-232. Recuperado de https://dusunenadamdergisi.org/article/216

I Papish, A., Kassam, A., Modgill, G., Vaz, G., Zanussi, L., y Patten, S. (2013). Reducir el estigma de las enfermedades mentales en la educación médica de pregrado: un ensayo controlado aleatorio. $B M C$ Educación médica, 13(141). Recuperado de https://pubmed.ncbi.nlm.nih.gov/24156397/

I Parra, L., Ortiz, N., y Moya, C. (2017). Efectos de la experiencia clínica en las actitudes de estudiantes de enfermería hacia las personas con discapacidad psiquiatrica. Ciencia y enfermería, 23(3) Recuperado de php?pid=S0717955 32017000300125\&script=sci_arttext\& tlng=en

I Pedersen, D. (2005). Estigma y exclusión social en las enfermedades mentales: apuntes para el análisis e investigación. Acta Psiquiátrica y Psicológica de América Latina, 55(1), 39-50. Recuperado de https:// www.researchgate.net /publication/281364699

I Petkari, E. (2017). Construyendo mentes hermosas: enseñar a través de películas para abordar el estigma en estudiantes de psicología en los Emiratos Árabes Unidos. Psiquiatría académica, 41, 724-732. Recuperado de https://doi.org/10.1007/s40596-017-0723-3

I Pingani, L., Coriani, S., Galeazzi, G., Nasi, A. M., y Franceschini, C. (2020). ¿Se puede prevenir las actitudes estigmatizantes en los estudiantes de psicología? Revista de salud mental, doi: 10.1080 / 09638237.2020.1714004

I Polanco-Frontera, N., Cajigas-Vargas, I., Rivera-Segarra, E., Varas-Díaz, N., Santos-Figueroa, A., Rosario-Hernández, E. (2013). Estigma hacia problemas de salud mental entre profesionales de la salud en adiestramiento en Puerto Rico. Salud y Sociedad, 4(3), 250-263. ISSN 0718-7475. 
I Poreddi, V., Thimmaiah, R., y BadaMath, S. (2017). Medical and nursing students attitudes toward mental illness: An Indian perspective. Investigación y Educación en Enfermería, 35(1), 86-94. doi.org/10.17533/ udea.iee.v35n1a10

I Pranckeviciene, A., Zardeckaite-Matulaitiene, K., Marksaityte, R., Endriulaitiene, A., Tillman, D., y Hof, D. (2018). Distancia social en estudiantes y profesionales de psicología y trabajo social lituanos. Psiquiatría social y epidemiología psiquiátrica, 53, 849-857. Recuperado de https://doi.org/10.1007/ s00127-018-1495-0

I Querido, A., Tomás, C., y Carvalho, D. (2016). O Estigma face á doenca mental nos estudantes de saúde. Revista Portuguesa de Enfermagem de Saúde Mental (Spe.3), 67-72. doi.org/10.19131/rpesm.0120

I Rodríguez-Meirinhos, A. y Antolín-Suárez (2020). Estigma social hacia la enfermedad mental: factores relacionados y propiedades psicométricas del Cuestionario de Atribuciones-revisado. Universitas Psychologica, vol. 19, 2020. Recuperado de https://revistas.javeriana.edu.co/index.php/revPsycho/article/ view/22117

I Sanz, J. (2018). Estigma de los profesionales de enfermería de salud mental hacia las personas con trastorno mental grave. Revista española de enfermería de salud mental, 7(2), 4-21. Recuperado de http:// www.reesme.com/index.php/REESM-AEESME/article/view/36

I Sastre, M. (2019). Construcción y validación de una escala para evaluación del estigma de la enfermedad mental en enfermería (tesis de doctorado) Recuperado de https://dialnet.unirioja.es/servlet/ tesis? codigo $=266031$

I Sawsan, A., Reem, H., Kimberly, H., y Muayyad, M. (2019). Revista de servicios de enfermería psicosocial y salud mental, 57(1), 43-51. Recuperado de https://doi.org/10.3928/02793695-20180907-01

I Senra-Rivera, C., Arriba-Roseto, A., y Seoane-Pesqueira, G. (2008). Papel de la experiencia en la aceptación vs. Rechazo del paciente con esquizofrenia. Revista Latinoamericana de Psicología, 40(1), 73-83. Recuperado de https://www.redalyc.org/pdf/805/80500106.pdf

I Simmons, L., Jones, T., y Bradley, E. (2017). Reducir el estigma de la salud mental. Revista europea de salud mental, 12(1), 25-40. Recuperado de https://psycnet.apa.org/record/2017-31380-002

I Tillman, D. T., Hof, D., Pranckeviciene, A., Endriulaitiené, A., Marksaityté, R., Zardeckaité-Matulaitiené, K. (2018). Distancia social de la enfermedad mental entre los estudiantes de consejería, trabajo social y psicología. Journal for social action in counseling and psychology, 10(1), 24-37. doi: https://doi. org/10.33043/JSACP.10.1.24-37

I Thornicroft, G. (2008). Stigma and discrimination limit access to mental health care. Epidemiologia E Psichiatria Sociale, 17(1), 14-9. Recuperado de https://scholarworks.uaeu.ac.ae/psych_theses/5

I Thornicroft, C., Wyllie, A., Thornicroft, G., y Mehta, N. (2013). Impact of the "Like Minds, Like Mine" anti-stigma and discrimination campaign in New Zealand on anticipated and experienced discrimination. Australian \& New Zealand Journal of Psychiatry, 48(4), 360-370. doi.org/10.1177/0004867413512687

I Thornicroft, G., Mehta, N., Clement, S., Evans-Lacko, S., Doherty, M., Rose, D., Koschorke, M., Shidhaye, R., O'Reilly, C., \& Henderson, C. (2016). Evidence for effective interventions to reduce mental-healthrelated stigma and discrimination. Lancet, 387(10023), 1123-1132. doi: 10.1016/S01406736 (15)00298-6. 
I Varga-Huicochea, I., Robles-García, R., Berlanda, C., Tovilla-Zárate, C., Martínez-López, N., y Fresan, A. (2017). Salud mental, 40(4), 141-147. Recuperado de https://doi.org/10.17711/sm.0185-3325.2017.018

I Waugh, W., Lethem, C., Sherring, S., y Henderson, C. (2017). Explorando experiencias y actitudes hacia la enfermedad mental y la divulgación entra los profesionales de la salud: un estudio cualitativo. Revista de salud mental, 26(5), 457-463. Recuperado de https://doi.org/10.1080/09638237.2017.1322184

I Wright, S., Henderson, C., Thornicroft, G., Sharac, J., y McCrone, P. (2015). Measuring the economic costs of discrimination experienced by people with mental health problems: development of the Costs of Discrimination Assessment (CODA). Social Psychiatry and Psychiatric Epidemiology, 50(5), 787-795. doi:10.1007/s00127-014-0968-z.

I Zaninotto, L., Qian, J., Sun, Y., Bassi, G., Solmi, M., y Salcuni, S. (2018). Género, rasgos de personalidad y experiencia con pacientes psiquiátricos como predictores de estigma en estudiantes italianos de psicología. Frontier in public health, 6: 362. doi: 103389/fpubh.2018.00362

I Zárate, C., Ceballos, M. E., Contardo, M. V. y Florenzano, R. (2006). Influencia de dos factores en la percepción hacia los enfermos mentales; contacto cercano y educación en salud. Revista chilena de neuropsiquiatría, 44(3), 205-214. doi.org/10.4067/S0717-92272006000300006

I Zhu, Y., Zhang, H., Yang, G., Hu, X., Liu, Z., Guo, N., He, H., Sun, B., y Rosenheck, R. (2018). Actitudes hacia las enfermedades mentales entre los estudiantes de medicina en China: impacto de la educación médica en el estigma. Asia-Pacific Psychiatry, 20(2), 1-7. Recuperado de https://doi.org/10.1111/ appy 12294

Envío a dictamen: 8 de febrero de 2021

Reenvío: 12 de mayo de 2021 Aprobación: 31 de mayo de 2021

Alma Alberta Martínez Castillo. Psicóloga social egresada de la Universidad Autónoma Metropolitana, con estudios de maestría en la Universidad Autónoma del Estado de México. Ha participado en diversos congresos nacionales como el XIV Congreso Mexicano de Psicología y, el 2do Congreso Internacional de Psicología de la Universidad Autónoma del Estado de México; también ha participado en el Congreso Internacional de Investigación y Practica Profesional en Psicología, Buenos Aires, Argentina. Cuenta con una publicación en la Revista de Psicología de la UAEMex La construcción del Sí mismo, de la mujer mixteca, desde la perspectiva de género". Ha colaborado en la publicación "Ansiedad, depresión y modos de afrontamiento en estudiantes pre universitarios" en la Revista de la Universidad de la Sierra Sur; y en la publicación "El estigma hacia 
los pacientes psiquiátricos: una revisión bibliográfica" de la Revista Alternativas en Psicología (por publicarse).Correo electrónico: aamc25103@hotmail.com

Roberto Lagunes Córdoba. Doctor en Ciencias. Miembro del Sistema Nacional de Investigadores. Ha participado como docente en la Escuela de Enfermería Naval de la Armada de México, en la Facultad de Medicina, en el Instituto de Salud Púbica y en el Instituto de Investigaciones Psicológicas de la Universidad Veracruzana. Además, ha dirigido de diversas tesis de licenciatura, especialidad, maestría y doctorado. Ha sido ponente en diversos congresos realizados en el país. Actualmente es Investigador de tiempo completo en el Instituto de Investigaciones Psicológicas, Universidad Veracruzana. Correo electrónico: rlc.academico@yahoo.com.mx

Francisco Javier Rosas Santiago. Doctor en Psicología. Ha participado como docente en diversas Universidades como: Universidad de la Sierra Sur de Oaxaca, Anáhuac Xalapa, Universidad Regional del Sureste, Instituto Mexicano de Psicoterapia Cognitivo Conductual, Instituto Cognitivo de Salud Mental, Universidad La Salle León y ha sido instructor del Diplomado en Diagnóstico y Tratamiento Cognitivo Conductual que se imparte en la Sociedad Mexicana de Psicología. Ha realizado estancias de investigación en: Instituto Nacional de Psiquiatría Ramón de la Fuente Muñiz, Hospital Psiquiátrico Fray Bernardino Álvarez, Centro Médico Nacional Siglo XX, Universidad Autónoma de Barcelona, Universidad de Barcelona y Universidad de Cádiz, España. Tiene más de 10 años de experiencia como investigador, docente y terapeuta. Ha sido ponente en diversas plataformas nacionales y extranjeras. Actualmente es investigador del Instituto de Investigaciones Psicológicas de la Universidad Veracruzana, donde preside el Comité de Ética en Investigación. Además, es miembro del Sistema Nacional de Investigadores. Correo electrónico: frosas98@yahoo.com.mx 